\title{
Across-site heterogeneity of genetic and environmental variances in the genetic evaluation of Eucalyptus globulus trials for height growth
}

\author{
João Costa E SILVA ${ }^{\mathrm{a} *}$, Gregory W. DuTKOWSKI ${ }^{\mathrm{b}}$, Nuno M.G. BorRALHO ${ }^{\mathrm{c}}$

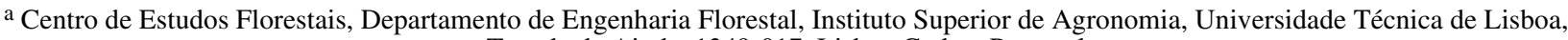 \\ Tapada da Ajuda, 1349-017 Lisboa Codex, Portugal \\ b Cooperative Research Centre for Sustainable Production Forestry, School of Plant Science, University of Tasmania, \\ Private Bag 55, Hobart 7001, Tasmania, Australia \\ ${ }^{c}$ RAIZ Forest and Paper Research Institute, Herdade da Torre Bela, Apartado 15, 2065 Alcoentre, Portugal
}

(Received 10 November 2003; accepted 9 June 2004)

\begin{abstract}
Height data from six 3-year-old Eucalyptus globulus trials with cloned progenies were jointly analysed with a heterogeneous variances model. Significant heterogeneity between trial sites was detected for additive genetic and environmental variances, corresponding to coefficients of variation of $41 \%$ and $26 \%$, respectively. Two additive genetic and four environmental variances were significantly different from common estimates across all trials. Significant heterogeneity was also detected for heritability estimates, which ranged from $13.5 \%$ to $40.3 \%$. Genetic evaluations of parents and clones within full-sib families were obtained from the heterogeneous variances model, and from a simpler model assuming variance homogeneity across trial sites and using either unadjusted data or data pre-adjusted by scale transformations. Changes in predictions of breeding values, top ranking genotypes and selection responses were examined to assess the impact of ignoring heterogeneous variances on the genetic evaluation. Clones were more sensitive than parents to the assumption of homogeneous variances in the evaluation model. Nevertheless, ignoring variance heterogeneity decreased the response to clonal selection by only $2 \%$ relatively to the evaluation based on the heterogeneous variances model. Pre-adjusting the data to constant phenotypic or environmental variances reduced the variance heterogeneity. The latter scale transformation was somewhat more effective in increasing fairness of selection, and resulted in close to optimal ranking and selection response. On the basis of the results of this study, Best Linear Unbiased Prediction was fairly robust to erroneously assuming homogeneous variances in a genetic evaluation model.
\end{abstract}

Eucalyptus globulus / heterogeneous variances / genetic evaluation / breeding values / selection

Résumé - Prise en compte de l'hétérogénéité inter-site des variances génétique et environnementale dans l'analyse d'essais d'Eucalyptus globulus pour la croissance en hauteur. Des mesures de hauteur, collectées à 3 ans dans 6 tests de descendances clonées d'Eucalyptus globulus, ont été analysées avec un modèle d'analyse de variance prenant en compte l'hétérogénéité des variances. Une hétérogénéité significative a été mise en évidence entre sites pour les variances génétiques additives et environnementales $(\mathrm{CV}=41 \%$ et $26 \%$ respectivement). Deux variances génétiques additives et quatre variances environnementales se sont révélées significativement différentes des variances estimées sur l'ensemble des sites. Une hétérogénéité significative a été aussi mise en évidence pour les héritabilités qui variaient de 13,5 à 40,3\%. Les valeurs génétiques des parents et des clones intra-familles de pleins-frères ont été estimées à partir d'un modèle d'analyse de variance prenant en compte cette hétérogénéité de variances et à partir d'un modèle simple d'analyse de variance assumant l'homogénéité des variances à travers sites, soit sur données non ajustées, soit sur données pré-ajustées par transformation scalaire. Les changements dans la prédiction des valeurs génétiques des génotypes, de leur classement et des réponses à la sélection ont été examinés pour établir l'impact de la non-prise en compte de ces variances hétérogènes sur l'estimation des paramètres génétiques. Les clones ont été plus sensibles que leurs parents par rapport à cette hypothèse de variances homogènes. Néanmoins, ignorer cette hétérogénéité de variances ne décroît la réponse à la sélection clonale que de $2 \%$ par rapport à une évaluation tenant compte de cette hétérogénéité. L'ajustement préalable des données à une constante phénotypique ou environnementale a réduit l'hétérogénéité des variances. La transformation à partir de la constante environnementale a été plus efficace en améliorant le classement des génotypes et la réponse à la sélection. Sur base des résultats de cette étude, le modèle linéaire mixte (BLUP) est apparu particulièrement robuste pour estimer les paramètres génétiques en assumant erronément l'homogénéité des variances.

Eucalyptus globulus / hétérogénéité des variances / évaluation génétique / valeur génétique / sélection

\footnotetext{
* Corresponding author: jces@isa.utl.pt
} 
Table I. Details of the examined Eucalyptus globulus field trials.

\begin{tabular}{|c|c|c|c|c|c|c|}
\hline Details & Trial 1 & Trial 2 & Trial 3 & Trial 4 & Trial 5 & Trial 6 \\
\hline \multicolumn{7}{|l|}{ Location and environment } \\
\hline Locality & Alcácer do Sal & Penalva do Castelo & Odemira & Castelo de Paiva & Azambuja & Ponte do Lima \\
\hline Latitude (North) & $38^{\circ} 22^{\prime}$ & $40^{\circ} 42^{\prime}$ & $37^{\circ} 25^{\prime}$ & $41^{\circ} 00^{\prime}$ & $39^{\circ} 06^{\prime}$ & $41^{\circ} 45^{\prime}$ \\
\hline Longitude (West) & $08^{\circ} 20^{\prime}$ & $07^{\circ} 39^{\prime}$ & $08^{\circ} 39^{\prime}$ & $08^{\circ} 23^{\prime}$ & $08^{\circ} 49^{\prime}$ & $08^{\circ} 29^{\prime}$ \\
\hline Altitude (m) & 50 & 550 & 300 & 150 & 70 & 400 \\
\hline Mean annual rainfall (mm) & 600 & 1400 & 1000 & 1400 & 600 & 2200 \\
\hline Mean annual temperature $\left({ }^{\circ} \mathrm{C}\right)$ & 15 & 12.5 & 15 & 12.5 & 16.5 & 12.5 \\
\hline \multicolumn{7}{|l|}{ Field layout } \\
\hline Number of replicates & 7 & 8 & 10 & 10 & 10 & 10 \\
\hline Spacing $(\mathrm{m} \times \mathrm{m})$ & $3.0 \times 3.0$ & $3.5 \times 2.0$ & $5.0 \times 2.0$ & $4.0 \times 1.9$ & $3.5 \times 1.8$ & $5.5 \times 1.8$ \\
\hline
\end{tabular}

\section{INTRODUCTION}

In recent years, there has been a considerable increase in the application of best linear unbiased prediction (BLUP, [12]) to evaluate genetic merit in forest tree breeding. Under the statistical linear mixed model, the properties of minimum prediction error variance and unbiasedness of the BLUP predictors of genetic value will hold if the variances and covariances of all the observations are properly specified and used in the evaluations. In practice, the exact covariance structure is not known, and the respective parameters need to be estimated. In addition, when data sets are large, models used in genetic evaluation may require as few fitted parameters as possible to allow computations to be feasible. Therefore, departures from the ideal model may occur, leading to predictions that only approximate BLUP.

One assumption often made in models of genetic evaluation using BLUP is that genetic and residual variances are homogeneous across environments. In animal breeding, several studies have shown that this assumption may not hold for production and conformation traits $[1,2,6,26,27,31]$. Also in tree breeding, different progeny tests are likely to have heterogeneous variances for growth traits, which may be due to different relative amounts of experimental error and/or scale effects associated with different ages or rates of growth at a given age [32]. Hill [14] indicated that variance heterogeneity results in selection of a greater fraction of individuals from the more variable environments. Therefore, when variance homogeneity is incorrectly assumed, there will be a tendency for overevaluating superior individuals in environments with large variances. This may reduce the response to selection when the heritability is greater in the less variable environments [7]. Accounting for heterogeneous variances may increase fairness of selection [1, 31 ], and improve predictions of breeding values by increasing accuracy and reducing bias [21, 26].

If reliable estimates of variances and covariances are available, the heterogeneity of variances across environments may be accounted for by using a multivariate model where the performance in each environment is considered as a separate trait $[8,13]$. However, computational demands and/or lack of appropriate parameter estimates may require the use of simpler models and, in this context, several adjustment methods based on scale transformations have been suggested to correct for variance heterogeneity. For example, assuming that genetic correlations across environments are close to one, the multivariate model mentioned above can be simplified to a univariate approach by adjusting the data to a common genetic scale, and heterogeneous residual variances and heritabilities are accounted for in the mixed model equations $[1,3,8,13]$. A simpler procedure is to assume homogeneous genetic and residual variances after data adjustments using approximations based on phenotypic variances (e.g. [2, 14, 27-30]), although it may not accommodate differences in heritability across environments. Scaling the data to a constant residual variance has also been applied to deal with variance heterogeneity $[5,15]$.

The objectives of this study were (1) to detect heterogeneity of additive genetic and environmental variances for 3-year-old height growth measured across six trial sites of Eucalyptus globulus ssp. globulus (hereafter referred to as E. globulus), and (2) to evaluate the impact of ignoring variance heterogeneity on the genetic evaluation. In the latter context, a model accounting for variance heterogeneity was compared with simpler analyses assuming constant variances and using either unadjusted data or observations pre-adjusted by scale transformations.

\section{MATERIALS AND METHODS}

\subsection{Plant material and field trials}

This study used 3-year-old height data from six E. globulus trials with clonally replicated progenies derived from controlled crosses. All base parents used in the crossings were from plus trees selected in commercial plantations in Portugal, and belong to the commonly referred Portuguese land race. The selection criteria were overall good growth and form, compared with immediate neighbour trees. Controlled crosses were carried out between 1992 and 1997 in the seed orchards of RAIZ (a Portuguese forest and paper research institute). Limitations in successfully completing the crosses led to a sparse diallel mating scheme. No reciprocals or selfs were attempted.

Table I provides general information about the field trials examined in this study. The trial sites correspond to contrasting growing conditions in Portugal. All trials used randomized block designs, and the number 
of replicates was 10 for most trials. Each full-sib family was normally represented in each replicate and, in order to provide a more efficient sampling, the clones within full-sib families were randomly allocated to single-tree non-contiguous plots within replicates. Details about the production of planting stock are described in Costa e Silva et al. [4].

The data comprised 45 parents, 92 full-sib families and 466 clones within full-sib families. The average numbers of crosses per parent and clones per full-sib family were 4 and 5, respectively. In terms of connectedness amongst trials, the number of common parents, full-sib families and clones varied between 26 and 37, 22 and 47, and 17 and 139 , respectively. The distribution of parents across trials (No. parents/ No. trials) was $26 / 6,5 / 5,1 / 4,5 / 3,4 / 2$ and $4 / 1$. For clones, the representation across trials (No. clones/No. trials) was 0/6, 29/5, 34/4, 76/ $3,106 / 2$ and $221 / 1$.

\subsection{Base model}

The following general linear mixed model was fitted to the data combined across the six trials:

$$
\mathbf{y}=\mathbf{X b}+\mathbf{Z}_{\mathbf{1}} \mathbf{a}+\mathbf{Z}_{\mathbf{2}} \mathbf{f}+\mathbf{Z}_{\mathbf{3}} \mathbf{c}+\mathbf{e}
$$

where $\mathbf{y}$ is a vector of observations on height growth, $\mathbf{b}$ is a vector of fixed effects (i.e. overall mean, trials and replicates within trials), $\mathbf{a}$ is a vector of random genetic effects of individual genotypes (i.e. parent trees and cloned progenies), $\mathbf{f}$ is a vector of random full-sib family effects, $\mathbf{c}$ is a vector of random effects of clones within full-sib families, and $\mathbf{e}$ is a vector of random residual terms. $\mathbf{X}, \mathbf{Z}_{\mathbf{1}}, \mathbf{Z}_{\mathbf{2}}$ and $\mathbf{Z}_{\mathbf{3}}$ are known incidence matrices relating the observations in $\mathbf{y}$ to effects in $\mathbf{b}, \mathbf{a}, \mathbf{f}$ and $\mathbf{c}$, respectively. The random effects in the model were assumed to follow a multivariate normal distribution with means and variances defined by:

$$
\left[\begin{array}{l}
\mathbf{a} \\
\mathbf{f} \\
\mathbf{c} \\
\mathbf{e}
\end{array}\right] \sim N\left(\left[\begin{array}{l}
\mathbf{0} \\
\mathbf{0} \\
\mathbf{0} \\
\mathbf{0}
\end{array}\right],\left[\begin{array}{llll}
\mathbf{G} & \mathbf{0} & \mathbf{0} & \mathbf{0} \\
\mathbf{0} & \mathbf{I}_{1} \sigma_{f}^{2} & \mathbf{0} & \mathbf{0} \\
\mathbf{0} & \mathbf{0} & \mathbf{I}_{2} \boldsymbol{\sigma}_{c}^{2} & \mathbf{0} \\
\mathbf{0} & \mathbf{0} & \mathbf{0} & \mathbf{R}
\end{array}\right]\right)
$$

where $\mathbf{0}$ is a null matrix, $\mathbf{G}$ is the (co)variance matrix of genetic effects for individual genotypes, $\mathbf{I}_{\mathbf{1}}$ and $\mathbf{I}_{\mathbf{2}}$ are identity matrices (with orders $f t$ and $c t$, respectively, where $f, c$ and $t$ are the numbers of full-sib families, clones and trials) and $\mathbf{R}$ is the residual variance matrix. For a model assuming variance heterogeneity across sites for effects in $\mathbf{a}$ and e (hereafter referred to as the base model or BM), $\mathbf{G}=(\mathbf{D} \mathbf{J} \mathbf{D}) \otimes$ $\mathbf{A}$ and $\mathbf{R}=\oplus_{i=1}^{6} \quad \mathbf{R}_{i}=\oplus_{i=1}^{6} \mathbf{I}_{n_{i}} \sigma_{e_{i}}^{2}$, where $\mathbf{D}$ is a diagonal matrix with elements equal to the square root of $\sigma_{a}^{2}$ at trial $i$ (i.e. $\sigma_{a}^{2}$, with $i$ $=1 \ldots 6), \mathbf{J}$ is a square matrix with all elements equal to $1, \mathbf{A}$ is the numerator relationship matrix [13], $\mathbf{I}_{n}$ is an identity matrix (with order equal to $n_{i}$, the number of trees at trial $i$ ), $\otimes$ is the Kronecker product and $\oplus$ is the direct sum operation. Observations on different ramets of a given clone were treated as repeated measurements on a single genotype, and thus $\mathbf{A}$ describes the additive genetic relationships among individual genotypes rather than among individual trees. $\sigma_{a}^{2}$, $\sigma_{f}^{2}, \sigma_{c}^{2}$ and $\sigma_{e}^{2}$ denote variance parameters, namely the genetic variance between individual genotypes, the variance between full-sib families, the variance among clones within full-sib families and the residual variance, respectively. Restricted maximum likelihood (REML, [23]) estimates of variance components and their standard errors were obtained by using the average information REML algorithm [9], implemented in the ASREML program [10]. The standard errors of the estimated parameters were calculated by the square root of the respective sampling variances, obtained from the inverse of the average information matrix. Using the estimated components of variance, approximations of additive genetic, dominance, epistatic and environmental variances may be obtained by $\hat{\sigma}_{a}^{2}, 4 \hat{\sigma}_{f}^{2}, \hat{\sigma}_{c}^{2}-3 \hat{\sigma}_{f}^{2}$ and $\hat{\sigma}_{e}^{2}$, respectively [4]. This derivation assumes that non-random environmental effects common to the ramets of a given clone (i.e. "C effects", [17]) are negligible, and that low-order interloci interactions represent a small portion of the total epistasis. Besides considering heterogeneous variances for effects in $\mathbf{a}$ and $\mathbf{e}$, the definition of BM assumes that:

(i) the correlations across sites for effects in $\mathbf{a}$ are homogeneous and equal to 1 ;

(ii) the effects in $\mathbf{f}$ are uncorrelated across sites and have a constant variance;

(iii) the effects in $\mathbf{c}$ are uncorrelated across sites and have a constant variance.

Previous bivariate analyses conducted for all pairs of trials, and accounting for variance heterogeneity across sites for effects in $\mathbf{a}, \mathbf{f}$, $\mathbf{c}$ and e, provided generally high correlations (i.e. range from 0.75 to 0.99 , with an average of 0.95 ) between sites for effects in $\mathbf{a}$. Moreover, combining the data over all trials and fitting heterogeneous variances for all effects, the REML log-likelihood ( $\operatorname{LogL}$ ) for a model constraining the across-site correlations for effects in a to be equal to $1 \mathrm{did}$ not differ substantially (i.e. 3.63) from the LogL obtained under an extended model considering the genetic expression at different sites as different traits. These results suggested that, for the given genetic material, age and sampled sites, the level of genotype by environment $(\mathrm{G} \times \mathrm{E})$ interaction may be low. Therefore, the assumption (i) seemed to be plausible.

All of the models mentioned above to assess the importance of $\mathrm{G} \times$ $E$ interaction applied a diagonal matrix to fit the effects in $\mathbf{f}$ and $\mathbf{c}$, which allows for variance heterogeneity while ignoring the covariances between sites for these effects. Previous single-site analyses, however, indicated very small and statistically non-significant variances for effects in $\mathbf{f}$ and $\mathbf{c}$. This suggested that across-site correlations for these effects would not be meaningful to estimate, and thus could be ignored. In addition, extending BM to incorporate heterogeneous variances for effects in $\mathbf{f}$ or $\mathbf{c}$ led to small improvements (i.e. 0.3 or 1.7, respectively) in LogL. Therefore, for the given genetic material and age, assumptions (ii) and (iii) seemed to be reasonable as a parsimonious solution to BM.

Given the components of variance estimated under BM, approximated estimates of heritability at each trial $\left(\hat{h}_{i}^{2}\right)$ were then obtained as follows:

$$
\hat{h}_{i}^{2} \approx \frac{\hat{\sigma}_{a_{i}}^{2}}{\hat{\sigma}_{a_{i}}^{2}+\hat{\sigma}_{f}^{2}+\hat{\sigma}_{c}^{2}+\hat{\sigma}_{e_{i}}^{2}}
$$

where $\hat{\sigma}_{a_{i}}^{2}$ and $\hat{\sigma}_{e_{i}}^{2}$ pertain to estimates at trial $i$, and $\hat{\sigma}_{f}^{2}$ and $\hat{\sigma}_{c}^{2}$ to pooled estimates. The sum of the variance components in the denominator of equation (3) estimates the phenotypic variance at trial $i\left(\hat{\sigma}_{p_{i}}^{2}\right)$. The standard errors of $\hat{\sigma}_{p_{i}}^{2}$ were obtained by the square root of the respective sampling variances, and these were calculated from the sampling (co)variances of the components in the denominator of equation (3). The standard errors of $\hat{h}_{i}^{2}$ were calculated according to the general expression for the variance of a ratio, based on an approximation using a first-order Taylor series expansion [19].

\subsection{Testing the homogeneity of variances across sites}

The homogeneity of $\hat{\sigma}_{a_{i}}^{2}$ and $\hat{\sigma}_{e_{i}}^{2}$ was tested via likelihood ratio(LR) tests. The test statistic $(D)$ was calculated by twice the difference between the LogL of BM, allowing for heterogeneous variances, and the $\log \mathrm{L}$ of a restricted model, which concerns the null hypothesis $\left(\mathrm{H}_{0}\right)$ to be tested. The magnitude of this difference indicates the strength of evidence against $\mathrm{H}_{0}$. Under $\mathrm{H}_{0}$, the distribution of $D$ asymptotically approximates that of a $\chi^{2}$, with degrees of freedom $(\mathrm{df})$ given by the difference between the number of parameters estimated 
under the BM and restricted models. The following LR tests were carried out sequentially:

(a) Overall test for homogeneity of both $\hat{\sigma}_{a_{i}}^{2}$ and $\hat{\sigma}_{e_{i}}^{2}$-the LogL under $\mathrm{H}_{0}$ is obtained by restricting both $\hat{\sigma}_{a_{i}}^{2}$ and $\hat{\sigma}_{e_{i}}^{2}$ to be homogeneous across sites; if the LR test is significant, then we may proceed with (b) and (c);

(b) Overall test for homogeneity of $\hat{\sigma}_{a}^{2}-$ the $\log \mathrm{L}$ under $\mathrm{H}_{0}$ is obtained by restricting $\hat{\sigma}_{a}^{2}$ to be equal while accounting for heterogeneous $\hat{\sigma}_{e_{i}}^{2}$; if the LR test is significant, then we may proceed with (d);

(c) Overall test for homogeneity of $\hat{\sigma}_{e}^{2}$ - the $\operatorname{LogL}$ under $\mathrm{H}_{0}$ is obtained by restricting $\hat{\sigma}_{e_{i}}^{2}$ to be equal while accounting for heterogeneous $\hat{\sigma}_{a_{i}}^{2}$; if the LR test is significant, then we may proceed with (e);

(d) Test an individual $\hat{\sigma}_{a_{i}}^{2}$ for deviation from the common additive genetic variance estimated in (b) - the $\operatorname{LogL}$ under $\mathrm{H}_{0}$ is obtained by restricting the given $\hat{\sigma}_{a_{i}}^{2}$ estimate to remain fixed at the common value;

(e) Test an individual $\hat{\sigma}_{e_{i}}^{2}$ for deviation from the common residual variance estimated in (c) - the $\log \mathrm{L}$ under $\mathrm{H}_{0}$ is obtained by restricting the given $\hat{\sigma}_{e_{i}}^{2}$ estimate to remain fixed at the common value.

In addition, the homogeneity of heritability across sites was also tested. This was carried out by following a procedure similar to (d), but using standardized data (to a constant phenotypic standard deviation of unity for each trial) instead of raw data, so that the estimated $\hat{\sigma}_{a_{i}}^{2}$ become equal to $\hat{h}_{i}^{2}$.

\subsection{Genetic evaluation from different models}

In order to assess the possible impact of variance heterogeneity on genetic evaluation, predictions of breeding values and selection outcomes were obtained from BM and from analyses assuming constant additive genetic and environmental variances (i.e. $\mathbf{G}=\mathbf{A} \sigma_{a}^{2}$ and $\mathbf{R}=$ $\left.\mathbf{I} \sigma_{e}^{2}\right)$. The aim was to determine whether the genetic evaluation based on a theoretically more correct model (i.e. BM) is substantially different from a simpler model assuming homogeneous variance. The simplified model used either unadjusted observations (hereafter referred to as evaluation E1), or data pre-adjusted by scale transformations (hereafter referred to as evaluations E2 or E3). The data adjustments applied to reduce the heterogeneity of variances were the scaling of the observations to constant phenotypic (E2) or environmental (E3) variances, using the following expression:

$$
y_{i j(a d j .)}=\left(y_{i j}-\bar{y}_{i}\right)+\frac{\hat{\sigma}_{p o p}}{\hat{\sigma}_{i}}+\bar{y}_{i}
$$

where $y_{i j(a d j .)}$ is the adjusted observation of ramet $j$ at trial $i, y_{i j}$ is the original observation of ramet $j$ at trial $i, \bar{y}_{i}$ is the height mean at trial $i$, $\hat{\sigma}_{\text {pop }}$ is the estimated (phenotypic or environmental) standard deviation for the population, and $\hat{\sigma}_{i}$ is the estimated (phenotypic or environmental) standard deviation at trial $i$. $\hat{\sigma}_{\text {pop. }}$. was calculated from the average of the $\hat{\sigma}_{p_{i}}^{2}$ or $\hat{\sigma}_{e_{i}}^{2}$ estimates across trials.

BLUPs of additive genetic values (i.e. predicted breeding values, PBV) for parents and cloned progeny were obtained from the base and simpler models, by solving the respective mixed model equations. Under BM, the PBVs were scaled to a common additive genetic variance by multiplying a PBV in trial $i$ by the ratio $\hat{\sigma}_{a_{(p o p)}} / \hat{\sigma}_{a_{i}}$, where $\hat{\sigma}_{a_{(p o p) .}}$ is the estimated additive genetic standard deviation for the population. For parents and clones represented at more than one trial, the (scaled) PBVs were then pooled. $\hat{\sigma}_{a_{(p o p}}$ was obtained from the average of the $\hat{\sigma}_{a}$ estimates, considering that the trials are all measured at the same age, assuming no $\mathrm{G} \times \mathrm{E}$ interaction and that no site is more representative of the plantation zone than any other. For complete and balanced data, this approach to yield evaluations for parents and clones from $\mathrm{BM}$ is equivalent to obtaining PBVs by using data adjusted to the same genetic scale and under an univariate model with heterogeneous residual variances and heritabilities accounted for in the mixed model equations (e.g. $[1,3,8,13])$.
Differences between predictions of breeding values from BM and $\mathrm{E} 1, \mathrm{E} 2$ or E3 were calculated for each genotype. The extent of possible bias in PBVs, caused by assuming variance homogeneity, was assessed by the average, maximum and minimum of the differences $\mathrm{BM}-\mathrm{E} 1, \mathrm{BM}-\mathrm{E} 2$ and BM - E3, between evaluations.

Parents and their cloned offspring were ranked on PBVs from BM and simpler models, and the best individuals (i.e. $25 \%$ of all parents and 5\% of all cloned offspring) were compared to evaluate the impact on selection of assuming variance homogeneity. Ranks of top individuals from BM were compared with ranks from the other evaluations (i.e. E1, E2 and E3) by the number of individuals in common, as well as the average and maximum rank change. The relative reduction in genetic gain due to ignoring variance heterogeneity was estimated by $\Delta_{G}=100\left(G_{S}-G_{B}\right) / G_{B}$, where $G_{B}$ and $G_{S}$ are expected genetic responses to selection under the base and simpler models, respectively. $G_{B}$ and $G_{S}$ were calculated as averages of PBVs for the individuals selected by each model, but using the predicted values from BM (i.e. the analysis assumed to be the most correct). As such, expected gains are obtained on the same scale, and differences between genetic responses reflect changes in the individuals selected.

\section{RESULTS}

Results from the overall LR tests are shown in Table II for unadjusted data and observations pre-adjusted by scale transformations. Tables III and IV present site parameter estimates obtained under BM and LR tests carried out at each individual trial for unadjusted and adjusted data, respectively. Table V presents a comparison of PBVs and selections from BM with PBVs and selections from the E1, E2 and E3 evaluations.

The height means varied between $7 \mathrm{~m}$ and $10 \mathrm{~m}$ across the trial sites (Tab. III). This range of mean values is comparable to that found in other progeny trials of E. globulus at a similar age (e.g. [16], for a large number of open-pollinated families tested across 5 sites in Tasmania).

As indicated by the model comparisons in Table II for unadjusted data, the overall LR tests of homogeneity detected significant differences $(P<0.001)$ among trials in $\hat{\sigma}_{a_{i}}^{2}$ and $\hat{\sigma}_{e_{i}}^{2}$. The $\hat{\sigma}_{a_{i}}^{2}$ and $\hat{\sigma}_{e_{i}}^{2}$ values ranged from 0.17 to 0.48 and from 0.66 to 1.49 (Tab. III), and had estimated across-site coefficients of variation $(\mathrm{CV})$ of $41 \%$ and $26 \%$, respectively. However, results from the overall LR tests (i.e. $D=146.88$ versus $D=23.62$, Tab. II) suggested that the heterogeneity of variances was most marked for $\sigma_{e_{i}}^{2}$. Accordingly, LR tests carried out for each trial (Tab. III) indicated that two $\hat{\sigma}_{a_{i}}^{2}$ and four $\hat{\sigma}_{e_{i}}^{2}$ estimates were significantly different $(P \leq 0.05)$ from common values (i.e. 0.26 and 1.09 , respectively) across sites. When judged in relation to the magnitude of the estimates, the standard errors for $\hat{\sigma}_{a_{i}}^{2}$ were larger than those for $\hat{\sigma}_{e_{i}}^{2}$ (Tab. III), which may have reduced the ability to distinguish between different $\hat{\sigma}_{a_{i}}^{2}$ estimates. Nevertheless, one-tailed LR tests [25] indicated that the $\hat{\sigma}_{a_{i}}^{2}$ values were significantly different $(P \leq 0.05)$ from zero for all trials (not shown). The pooled $\hat{\sigma}_{f}^{2}$ and $\hat{\sigma}_{c}^{2}$ were low (i.e. 0.02 and 0.03 , respectively) compared with $\hat{\sigma}_{a_{i}}^{2}$ and $\hat{\sigma}_{e_{i}}^{2}$. $\hat{\sigma}_{p_{i}}^{2}$ ranged from 1.19 to 1.81 (Tab. III), and had an estimated $\mathrm{CV}^{i}$ of $18 \%$.

The $\hat{h}_{i}^{2}$ values ranged from 0.135 to 0.403 (Tab. III), and had an estimated CV of $49 \%$. The range of $\hat{h}_{i}^{2}$ in this study approached that reported by Lopez et al. [18] in a review of genetic parameters 
Table II. Overall likelihood ratio tests for detecting variance heterogeneity in additive genetic $\left(\hat{\sigma}_{a_{i}}^{2}\right)$ and environmental $\left(\hat{\sigma}_{e_{i}}^{2}\right)$ variance estimates, obtained for 3-year-old height growth measured across six Eucalyptus globulus trials $(i=1, \ldots, 6)$. The analyses used unadjusted data (E1), or observations pre-adjusted by scaling to common phenotypic (E2) or environmental variances (E3). The tested null hypotheses $\left(\mathrm{H}_{0}\right)$, inherent to the restricted models, were: (1) $\hat{\sigma}_{a_{i}}^{2}$ and $\hat{\sigma}_{e_{i}}^{2}$ are both homogeneous over trial sites; (2) $\hat{\sigma}_{a_{i}}^{2}$ is homogeneous over trial sites; (3) $\hat{\sigma}_{e_{i}}^{2}$ is homogeneous over trial sites. Under $\mathrm{H}_{0}$, the distribution of the test statistic $(D)$ asymptotically approximates that of a $\chi^{2}$, with degrees of freedom (df) given by the difference between the number of parameters estimated under the base model and restricted models.

\begin{tabular}{|c|c|c|c|c|}
\hline & \multirow[t]{2}{*}{ Base model } & \multicolumn{3}{|c|}{ Restricted models } \\
\hline & & (1) & (2) & (3) \\
\hline \multicolumn{5}{|l|}{ E1 } \\
\hline $\log \mathrm{L}$ & $-3608.93^{b}$ & -3691.95 & -3620.74 & -3682.37 \\
\hline $\begin{array}{c}D^{\mathrm{a}} \\
\text { (df, } P \text {-value) }\end{array}$ & & $\begin{array}{c}166.04 \\
(10 \mathrm{df}, P<0.001)\end{array}$ & $\begin{array}{c}23.62 \\
(5 \mathrm{df}, P<0.001)\end{array}$ & $\begin{array}{c}146.88 \\
(5 \mathrm{df}, P<0.001)\end{array}$ \\
\hline \multicolumn{5}{|l|}{ E2 } \\
\hline $\log L$ & $-3626.34^{b}$ & 3656.81 & nt & $\mathrm{nt}$ \\
\hline $\begin{array}{c}D^{\mathrm{a}} \\
\text { (df, } P \text {-value) }\end{array}$ & & $\begin{array}{c}60.94 \\
(10 \mathrm{df}, P<0.001)\end{array}$ & nt & $\mathrm{nt}$ \\
\hline \multicolumn{5}{|l|}{ E3 } \\
\hline $\log \mathrm{L}$ & $-3613.05^{\mathrm{c}}$ & $\mathrm{nt}$ & -3640.64 & nt \\
\hline $\begin{array}{c}D^{\mathrm{a}} \\
\text { (df, } P \text {-value) }\end{array}$ & & $\mathrm{nt}$ & $\begin{array}{c}55.18 \\
(5 \mathrm{df}, P<0.001)\end{array}$ & $\mathrm{nt}$ \\
\hline
\end{tabular}

nt: not tested.

a $D=2\left(\mathrm{~L}_{\mathrm{BM}}-\mathrm{L}_{\mathrm{RM}}\right)$, where $\mathrm{L}_{\mathrm{BM}}$ and $\mathrm{L}_{\mathrm{RM}}$ refer to the log-likelihoods (LogL) for the base and restricted models, respectively.

b Model allowing for variance heterogeneity in both $\hat{\sigma}_{a_{i}}^{2}$ and $\hat{\sigma}_{e_{i}}^{2}$.

${ }^{c}$ Model allowing for variance heterogeneity in $\hat{\sigma}_{a_{i}}^{2}$.

Table III. Individual trial estimates of means, additive genetic $\left(\hat{\sigma}_{a_{i}}^{2}\right)$, environmental $\left(\hat{\sigma}_{e_{i}}^{2}\right)$ and phenotypic $\left(\hat{\sigma}_{p_{i}}^{2}\right)$ variances, and heritabilities $\left(\hat{h}_{i}^{2}\right)$, for 3-year-old height growth measured across six Eucalyptus globulus trials $(i=1, \ldots, 6)$. For each trial, $D_{1}, D_{2}$ or $D_{3}$ are calculated test statistics from likelihood ratio tests, carried out for detecting whether an individual $\hat{\sigma}_{a_{i}}^{2}, \hat{\sigma}_{e_{i}}^{2}$ or $\hat{h}_{i}^{2}$ significantly $(P \leq 0.05)$ deviates from a common estimate over all trials. Under $\mathrm{H}_{0}$, the distribution of $D_{1}, D_{2}$ or $D_{3}$ asymptotically approximates that of a $\chi^{2}$, with one degree of freedom. The approximate standard errors for the estimated parameters are given in parenthesis.

\begin{tabular}{|c|c|c|c|c|c|c|c|c|}
\hline Trial $i$ & Mean (m) & $\hat{\sigma}_{a_{i}}^{2 \mathrm{a}}$ & $D_{1}$ & $\hat{\sigma}_{e_{i}}^{2}$ & $D_{2}$ & $\hat{\sigma}_{p_{i}}^{2}$ & $\hat{h}_{i}^{2}$ & $D_{3}$ \\
\hline 1 & 9.9 & $\begin{array}{c}0.48 \\
(0.08)\end{array}$ & $\begin{array}{c}9.66 \\
(P=0.002)\end{array}$ & $\begin{array}{c}0.66 \\
(0.03)\end{array}$ & $\begin{array}{c}80.9 \\
(P<0.001)\end{array}$ & $\begin{array}{c}1.19 \\
(0.09)\end{array}$ & $\begin{array}{l}0.403 \\
(0.05)\end{array}$ & $\begin{array}{c}15.04 \\
(P<0.001)\end{array}$ \\
\hline 2 & 8.0 & $\begin{array}{c}0.17 \\
(0.04)\end{array}$ & $\begin{array}{c}3.74 \\
(P=0.05)\end{array}$ & $\begin{array}{c}1.04 \\
(0.05)\end{array}$ & $\begin{array}{c}0.96 \\
(P>0.05)\end{array}$ & $\begin{array}{c}1.26 \\
(0.06)\end{array}$ & $\begin{array}{l}0.135 \\
(0.03)\end{array}$ & $\begin{array}{c}2.36 \\
(P>0.05)\end{array}$ \\
\hline 3 & 7.1 & $\begin{array}{c}0.34 \\
(0.07)\end{array}$ & $\begin{array}{c}1.30 \\
(P>0.05)\end{array}$ & $\begin{array}{c}1.26 \\
(0.06)\end{array}$ & $\begin{array}{c}9.1 \\
(P=0.003)\end{array}$ & $\begin{array}{c}1.65 \\
(0.09)\end{array}$ & $\begin{array}{l}0.206 \\
(0.04)\end{array}$ & $\begin{array}{c}0.08 \\
(P>0.05)\end{array}$ \\
\hline 4 & 8.3 & $\begin{array}{c}0.18 \\
(0.05)\end{array}$ & $\begin{array}{c}2.54 \\
(P>0.05)\end{array}$ & $\begin{array}{c}0.97 \\
(0.05)\end{array}$ & $\begin{array}{c}4.86 \\
(P=0.028)\end{array}$ & $\begin{array}{c}1.20 \\
(0.07)\end{array}$ & $\begin{array}{l}0.150 \\
(0.04)\end{array}$ & $\begin{array}{c}0.96 \\
(P>0.05)\end{array}$ \\
\hline 5 & 8.5 & $\begin{array}{c}0.27 \\
(0.07)\end{array}$ & $\begin{array}{c}0.0 \\
(P>0.05)\end{array}$ & $\begin{array}{c}1.49 \\
(0.07)\end{array}$ & $\begin{array}{c}49.46 \\
(P<0.001)\end{array}$ & $\begin{array}{c}1.81 \\
(0.09)\end{array}$ & $\begin{array}{l}0.149 \\
(0.03)\end{array}$ & $\begin{array}{c}1.34 \\
(P>0.05)\end{array}$ \\
\hline 6 & 8.5 & $\begin{array}{c}0.25 \\
(0.05)\end{array}$ & $\begin{array}{c}0.06 \\
(P>0.05)\end{array}$ & $\begin{array}{c}1.05 \\
(0.04)\end{array}$ & $\begin{array}{c}1.02 \\
(P>0.05)\end{array}$ & $\begin{array}{c}1.35 \\
(0.06)\end{array}$ & $\begin{array}{l}0.185 \\
(0.03)\end{array}$ & $\begin{array}{c}0.02 \\
(P>0.05)\end{array}$ \\
\hline
\end{tabular}

a Additive genetic variance estimates were all significantly $(P \leq 0.05)$ different from zero.

calculated for height at ages 3 and 4 years in E. globulus. However, as shown in Table III, in only one case was the heritability significantly different $(P<0.001)$ from a common estimate (i.e. 0.19). This result reflected the rather high heritability (i.e. 0.403 ) at trial 1 versus a group of trials with modest differences in $\hat{h}_{i}^{2}$ (Tab. III), suggesting that the heritability estimates within the range from 0.135 to 0.206 may not be significantly different from each other. The estimated standard errors of the $\hat{h}_{i}^{2}$ were not extremely large relative to the parameter estimates themselves (Tab. III). Nevertheless, for the narrow range from 0.135 to 0.206 , more parents and progeny per parent would have been required to increase the statistical power of the LR tests and, thereby, the chances to detect true differences for the $\hat{h}_{i}^{2}$ estimates. 
Table IV. Effects of scale transformations on site estimates of additive genetic $\left(\hat{\sigma}_{a_{i}}^{2}\right)$ and environmental $\left(\hat{\sigma}_{e_{i}}^{2}\right)$ variances, obtained for 3-year-old height growth measured across six Eucalyptus globulus trials $(i=1 \ldots 6)$. The data were pre-adjusted by scaling to constant phenotypic or environmental variances. For each trial, $D_{1}$ or $D_{2}$ are calculated test statistics from likelihood ratio tests, carried out for detecting whether an individual $\hat{\sigma}_{a_{i}}^{2}$ or $\hat{\sigma}_{e_{i}}^{2}$ significantly $(P \leq 0.05)$ deviates from a common estimate over all trials. Under $\mathrm{H}_{0}$, the distribution of $D_{1}$ or $D_{2}$ asymptotically approximates that of a $\chi^{2}$, with one degree of freedom.

\begin{tabular}{|c|c|c|c|c|c|c|c|c|}
\hline \multirow[t]{2}{*}{ Trial $i$} & \multicolumn{5}{|c|}{$\begin{array}{l}\text { Scaling to constant } \\
\text { phenotypic variance }\end{array}$} & \multicolumn{3}{|c|}{$\begin{array}{l}\text { Scaling to constant } \\
\text { environmental variance }\end{array}$} \\
\hline & $\hat{\sigma}_{a_{i}}^{2}$ & $D_{1}$ & $\hat{\sigma}_{e_{i}}^{2}$ & $D_{2}$ & $\hat{\sigma}_{p_{i}}^{2}$ & $\hat{\sigma}_{a_{i}}^{2}$ & $D_{1}$ & $\hat{\sigma}_{p_{i}}^{2}$ \\
\hline 1 & 0.58 & $\begin{array}{c}15.46 \\
(P<0.001)\end{array}$ & 0.78 & $\begin{array}{c}34.74 \\
(P<0.001)\end{array}$ & 1.41 & 0.80 & $\begin{array}{c}33.88 \\
(P<0.001)\end{array}$ & 1.92 \\
\hline 2 & 0.19 & $\begin{array}{c}2.26 \\
(P>0.05)\end{array}$ & 1.17 & $\begin{array}{c}3.18 \\
(P>0.05)\end{array}$ & 1.41 & 0.17 & $\begin{array}{c}3.30 \\
(P>0.05)\end{array}$ & 1.29 \\
\hline 3 & 0.28 & $\begin{array}{c}0.06 \\
(P>0.05)\end{array}$ & 1.08 & $\begin{array}{c}0.0 \\
(P>0.05)\end{array}$ & 1.41 & 0.28 & $\begin{array}{c}0.08 \\
(P>0.05)\end{array}$ & 1.40 \\
\hline 4 & 0.21 & $\begin{array}{c}0.96 \\
(P>0.05)\end{array}$ & 1.15 & $\begin{array}{c}1.30 \\
(P>0.05)\end{array}$ & 1.41 & 0.19 & $\begin{array}{c}1.48 \\
(P>0.05)\end{array}$ & 1.31 \\
\hline 5 & 0.20 & $\begin{array}{c}1.22 \\
(P>0.05)\end{array}$ & 1.16 & $\begin{array}{c}2.64 \\
(P>0.05)\end{array}$ & 1.41 & 0.19 & $\begin{array}{c}1.92 \\
(P>0.05)\end{array}$ & 1.31 \\
\hline 6 & 0.26 & $\begin{array}{c}0.0 \\
(P>0.05)\end{array}$ & 1.10 & $\begin{array}{c}0.18 \\
(P>0.05)\end{array}$ & 1.41 & 0.25 & $\begin{array}{c}0.02 \\
(P>0.05)\end{array}$ & 1.37 \\
\hline
\end{tabular}

As shown in Table III, the phenotypic variances increased with $\hat{\sigma}_{e_{j}}^{2}$, and $\hat{h}_{i}^{2}$ tended to be positively associated with $\hat{\sigma}_{a_{i}}^{2}$. Trial 1 had the highest levels for the additive genetic variance and heritability, and the lowest levels for environmental and phenotypic variances.

Significant variance heterogeneity was still detected after scaling the data to constant phenotypic or environmental variances, as indicated by results from the overall LR tests shown in Table II (i.e. $D=60.94$ and $D=55.18, P<0.001$ ). Nevertheless, the heterogeneity of $\hat{\sigma}_{e_{i}}^{2}$ was substantially reduced after the scale transformation to a constant phenotypic variance, as in only one case was $\hat{\sigma}_{e_{i}}^{2}$ significantly different $(P<0.001)$ from a common estimate (Tab. IV). For $\hat{\sigma}_{a_{i}}^{2}$, however, the data adjustment methods were less effective in reducing the heterogeneity of variances, although only one estimate remained significantly different $(P<0.001)$ from a common value (Tab. IV). Comparing Tables III and IV, the largest changes in $\hat{\sigma}_{a_{i}}^{2}$ and $\hat{\sigma}_{e_{i}}^{2}$ following the data adjustments were observed in trials 1 (i.e. +0.10 and +0.32 ) and 5 (i.e. -0.33 ), respectively.

Table $\mathrm{V}$ indicates that the analysis assuming homogeneous variances and using unadjusted data (i.e. E1) has, on average, overestimated the PBVs when compared with predictions from BM. Although the average difference between PBVs was small (i.e. $8 \mathrm{~mm}$ ), ignoring variance heterogeneity has overpredicted the breeding values from BM up to $297 \mathrm{~mm}$. Scaling the data to a constant environmental variance (i.e. E3) reduced somewhat the range of differences between PBVs and decreased the average bias to $4 \mathrm{~mm}$ (i.e. an improvement of $50 \%$ relatively to E1). Compared with E3, the scale transformation to an equal phenotypic variance (i.e. E2) was less effective in reducing both the average and range of differences between PBVs.

Ignoring the heterogeneity of variances had only a small effect on the ranks of top parents: as indicated in Table $\mathrm{V}$, the lists of the best 11 parents from $\mathrm{BM}$ and $\mathrm{E} 1$ had 10 in common, and the average and maximum PBV rank change between the two evaluations were only 0.5 and 3 , respectively. Compared with E1, the data adjustments under E2 and E3 did not cause major differences among the ranks of top parents. The evaluation of top clones, however, was more sensitive to variance heterogeneity, although the lists of the best 23 clones from BM and E1 had 19 in common, and the average and maximum PBV rank change between the two evaluations were modest (Tab. V). Scaling the data reduced somewhat the impact of ignoring variance heterogeneity on the clonal evaluation, with E3 resulting in a better agreement with the ranks of top clones from BM.

In terms of selection response, ignoring the heterogeneity of variances in E1 is expected to decrease the genetic progress from clonal selection by only $2.1 \%$ relatively to BM (Tab. V). Yet, the relative decrease in genetic progress under E1 is expected to be three times larger than that for E3 (i.e. $2.1 \%$ versus $0.7 \%$ ). For parental selection, ignoring variance heterogeneity under E1 led to a smaller reduction in genetic response when compared with the cloned progeny evaluations (i.e. $1.1 \%$ versus $2.1 \%$ ), and correction for heterogeneity under E2 and E3 did not change further the relative genetic response.

\section{DISCUSSION}

\subsection{Impact of ignoring heterogeneous variances on the genetic evaluation}

When the covariance structure is correctly specified, the BLUP analysis accounts for variance heterogeneity by properly weighting and scaling the data. Failure to account for variance heterogeneity from different environments may result in an incorrect model fitted to the data. This may lead to inaccurate 
Table V. Comparison of breeding value predictions and selections from the model allowing for heterogeneous $\hat{\sigma}_{a_{i}}^{2}$ and $\hat{\sigma}_{e_{i}}^{2}$ (i.e. base model, BM) with breeding value predictions and selections from the model assuming homogeneous $\hat{\sigma}_{a_{i}}^{2}$ and $\hat{\sigma}_{e_{i}}^{2}$. The latter model used unadjusted data (E1), or observations pre-adjusted by scaling to constant phenotypic (E2) or environmental (E3) variances. Differences (i.e. BM-E1, BM-E2 and BM-E3) between predictions of breeding values from alternative genetic evaluations were calculated for each individual, and the average, maximum and minimum of these differences are presented. Selections from alternative genetic evaluations were compared for top ranking parents (approx. 25\% selected) and clones (5\% selected). $\Delta_{G}$ is the estimated relative reduction in genetic response due to ignoring variance heterogeneity in the genetic evaluations E1, E2 and E3.

\section{BM vs. E1 BM vs. E2 BM vs. E3}

Breeding value predictions

$\begin{array}{lccc}\text { Average difference (m) } & -0.008 & -0.007 & -0.004 \\ \text { Maximum difference (m) } & -0.297 & -0.282 & -0.241 \\ \text { Minimum difference (m) } & 0.188 & 0.203 & 0.156\end{array}$

Selection

Parents $($ No. selected $=11)$

\begin{tabular}{lccc} 
No. in common & 10 & 10 & 10 \\
Average rank change & 0.5 & 0.7 & 0.8 \\
Maximum rank change & 3 & 3 & 5 \\
$\Delta_{G}(\%)$ & -1.1 & -1.3 & -1.3 \\
Clones (No. selected = 23) & & & \\
No. in common & 19 & 21 & 22 \\
Average rank change & 3.4 & 2.6 & 2.4 \\
Maximum rank change & 18 & 12 & 7 \\
$\Delta_{G}(\%)$ & -2.1 & -1.2 & -0.7 \\
\hline
\end{tabular}

and biased predictions of genetic values, and thus may affect selection decisions and estimates of genetic progress. Nevertheless, the assumption of variance homogeneity may be required for practical reasons in a model used for genetic evaluation. In this sense, it may be useful to assess whether the efficiency of selection based on simplified models assuming variance homogeneity is substantially altered when compared with a (more appropriate) heterogeneous variances model.

In this study, the across-site heterogeneity of additive genetic and environmental variance estimates was statistically significant. Therefore, correction for heterogeneous variances may be needed if a method assuming variance homogeneity is applied for breeding value evaluation. There was not a clear relationship between the trial means and the estimated variances (Tab. III), and thus the heterogeneity of variances can not simply be explained by a scale effect. $\hat{\sigma}_{e_{i}}^{2}$ may vary amongst trials because of real differences in microsite environmental effects and/or differing efficiency of the trial experimental designs to account for within-site environmental heterogeneity. The effect of sampling variability may be one possible cause for the heterogeneous $\hat{\sigma}_{a}^{2}$, as the trials differed in parental samples from the same base population and the number of tested parents was limited. Related to this possibility, heterogeneous $\sigma_{a_{i}}^{2}$ may also reflect different changes in additive genetic variance due to differences between parental samples in gametic disequilibrium generated by plus-tree selection for growth.

Using untransformed data, the model assuming variance homogeneity for $\hat{\sigma}_{a_{i}}^{2}$ and $\hat{\sigma}_{e_{i}}^{2}$ tended to overestimate the PBVs compared with the BM model (see maximum difference between BM and E1, Tab. V), although the estimated bias was not large. The biases in the genetic evaluation from assuming homogeneous variances had a little effect on the parental ranking. The majority of the parents were well represented across trials, and thus progeny in small variance sites may offset progeny in large variance sites. Yet the progeny of some parents were poorly distributed over different trials, and thus variance heterogeneity could potentially have had a greater impact on the parental evaluation. The offspring evaluation, however, was more sensitive (i.e. larger changes in ranking) than parental evaluation to violations of the assumed variance homogeneity. This is probably due to the fact that the distribution of cloned progenies across trials was highly unbalanced, with nearly $50 \%$ of the clones being tested in one site only. A clone with all of its measurements in a single trial is likely to be unfairly assessed if homogeneous variances are incorrectly assumed, which may reduce the efficiency of selection.

As shown in Table $\mathrm{V}$, four top ranking clones selected under E1 were not present in the list of selected clones from BM. The first three of these clones are poorly distributed across trials (i.e. with a representation at one or two sites) and are all tested at trial 5, where $\hat{\sigma}_{p_{i}}^{2}$ had the greatest value. This suggests that assuming homogeneous variances favoured the selection of clones tested in the most variable environment. In this case, the most variable site is also one of the least accurate for evaluations (i.e. with the greatest $\hat{\sigma}_{e_{i}}^{2}$ and a rather low $\hat{h}_{i}^{2}$, Tab. III), which may have contributed to reduce the expected genetic gain from clonal selection relative to the BM evaluation. However, although the ranks of the individual clones did change, most of the top ranking clones selected in BM were also selected in E1, and thus ignoring variance heterogeneity did not reduce substantially (i.e. $2 \%$ ) the selection response relative to $\mathrm{BM}$. The effects of ignoring variance heterogeneity on selection response depend on the differences in $\hat{h}_{i}^{2}$ among environments and their relation to $\hat{\sigma}_{p_{j}}^{2}[14,28]$. In this sense, the greatest reduction in gain from selection results when differences in heritability values across environments are strongly negatively correlated with changes in phenotypic variances [7]. Although the relationship between $\hat{h}_{i}^{2}$ and $\hat{\sigma}_{p \text {. }}^{2}$ seemed to be weak in the present study, there was still a possibility for a potential reduction in selection response due to erroneously assuming variance homogeneity, as there were sites with high $\hat{h}_{i}^{2}$ and low $\hat{\sigma}_{p_{i}}^{2}(\mathrm{e} . \mathrm{g}$. trial 1) and sites with low $\hat{h}_{i}^{2}$ and high $\hat{\sigma}_{p_{i}}^{2}(\mathrm{e}$.g. trial 5). Nevertheless, using a parameter set where the heritabilities decreased with increased phenotypic variances, Garrick and Van Vleck [7] reported a reduction in the rate of selection response of only $3 \%$ when variance heterogeneity was ignored in the genetic evaluation. Meuwissen and Van der Werf [20] also reported that ignoring heterogeneous variances between environments did not cause substantial losses in genetic gain. 
It could be argued that offspring selection based on net PBVs (e.g. Tab. V) may be less sensitive to ignoring variance heterogeneity than within-family selection. This is because the influence of parental PBVs on net offspring PBV s could dampen any changes in them compared with within-family breeding values (which are based on the part of the net offspring PBVs that is independent of the parental PBVs). However, in our data, the reduction in genetic response from ignoring variance heterogeneity was similar for offspring selections based on net PBVs and within-family breeding values. Predictions of within-family breeding values for cloned offspring are expected to be more accurate than for uncloned offspring, and thus the stabilizing effect of the parental breeding value contribution is likely to be diminished.

\subsection{Effects of data adjustments to reduce the heterogeneity of variances}

Although it was mainly due to a single estimate (i.e. 0.403 at trial 1), the heterogeneity in $\hat{h}_{i}^{2}$ values was significant and, as expected, was robust to the scale transformations used in this study. Consequently, the applied data adjustments could not remove all of the heterogeneity of variance, as this would require constant heritabilities across trials. Moreover, scaling the data to common phenotypic or environmental variances, while incorrectly assuming constant heritabilities, may not improve the selection efficiency compared with an evaluation where the heterogeneity of variances is ignored. This may occur when the heritability is higher in the more variable environments, in which case observations from low heritability environments will have more weight after scaling the data. In such a situation, a method of data adjustment accounting for heterogeneity in both variances and heritabilities may be appropriate [6]. Nevertheless, the heterogeneity in $\hat{h}_{i}^{2}$ values was associated with the heterogeneity of $\hat{\sigma}_{a,}^{2}$, but did not appear to be closely correlated with differing $\hat{\sigma}_{p_{i}}^{2}$ and $\hat{\sigma}_{e_{i}}^{2}$ across trials (Tab. III). Thus, the possible reduction in accuracy of the evaluations, from ignoring different $\hat{h}_{i}^{2}$ when scaling the data, is likely to be unimportant in the present study. In fact, trials with higher $\hat{h}_{i}^{2}$ were given more weight in the evaluation E3, which used data scaled to a constant environmental variance. This is because, following the data adjustment under E3, the magnitude of $\hat{\sigma}_{p_{i}}^{2}$ tended to become directly related to the $\hat{\sigma}_{a_{i}}^{2}$ estimates (Tab. IV) and, thereby, also to the $\hat{h}_{i}^{2}$ values.

The correction for variance heterogeneity decreased only partly the estimated biases in the PBVs, with the improvement being better after scaling to a constant environmental variance. Adjusting for variance heterogeneity had practically no effect on parental selection. However, the rankings of offspring clones were improved by consideration of variance heterogeneity, suggesting that some biases in the offspring evaluation could be corrected by the applied scale transformations. In particular, scaling the data to a constant environmental variance reduced the evaluations for top clones in the most variable site (i.e. trial 5), and resulted in close to optimal clonal ranking and selection response. The three top clones mentioned previously, and selected under E1, were not present in the list of selected clones from E3, as they were replaced by genotypes that are fairly well distributed across trials (i.e. at least in four sites) and are all tested at trial 1 , where $\hat{h}_{i}^{2}$ had the greatest value.

\subsection{Final considerations}

A full multivariate approach, where the performance in each environment is considered a separate trait, would be theoretically the best base model. However, as estimated correlations between trials were generally high for effects in a, the losses in accuracy due to assuming unit across-site genetic correlations in the base model are expected to be small. Garrick and Van Vleck [7] found a negligible effect on the efficiency of selection from assuming a unit genetic correlation across environments when in reality a small $\mathrm{G} \times \mathrm{E}$ interaction was present (as given by genetic correlations ranging from 0.86 to 0.97 ).

The applied base model allowed heterogeneous additive genetic variances to be more easily incorporated than in Griffing's model [11] for diallel mating designs, and extended to include cloned progenies [22, 24]. This is because, in Griffing's model, the variance due to general combining ability of the parents and the variance due to differences among clones within full-sib families both contain portions of the additive genetic variance. In addition, by using cloned progenies, the residual variance in the base model may be heterogeneous because of environmental effects, and not because of unaccounted for non-additive genetic variance (as by using seedling progenies). The base model also incorporated information (such as genetic relationships among genotypes) across trials, which may have contributed to increase the accuracy of variance components and heritabilities estimated for each site. Nevertheless, the validity of the applied base model for providing correct predictions of breeding values (and thus be used as a basis for determining the optimal genetic response to selection) is dependent on how well the estimated parameters approximate the true (co)variance structure.

\section{CONCLUSION}

The present study indicated that ignoring variance heterogeneity in the genetic evaluation may favour the selection of superior genotypes in the more variable sites, particularly if the individuals in question are poorly represented over different environments. In this context, clones within full-sib families were more sensitive than parents to the assumption of homogeneous variances in an evaluation model. As the more variable site in this study had also a low heritability, assuming homogeneous variances reduced the expected genetic gain from clonal selection relative to an evaluation accounting for heterogeneity of variances. However, this reduction in selection response was not substantial, which suggests that the BLUP method was reasonably robust to violations of assumptions regarding the homogeneity of variances across sites.

Adjusting the data to constant phenotypic or environmental variances removed some of the variance heterogeneity. Under the relationships among the parameters estimated in this study, scaling the data to a constant environmental variance was somewhat more effective in increasing fairness of selection, and resulted in close to optimal ranking and selection response.

While our data is representative of the range of tree sizes that have been found in other progeny trials of this species at a similar age [16], our conclusions may not apply if a wider range of variances exists due to different ages or productivities at a 
given age. In addition, our results were obtained from a series of sites which exhibited a low genotype by environment interaction and largely the same genetic material.

Acknowledgements: We wish to express our gratitude to Maria Helena Almeida for valuable discussions and support during this work, as well as to Brad Potts, Luis Apiolaza and two anonymous reviewers for helpful comments on the manuscript. We also thank José Alexandre Araújo for technical assistance with the field trials, and to Fundação para a Ciência e Tecnologia (Lisboa, Portugal) for financial support.

\section{REFERENCES}

[1] Boldman K.G., Freeman A.E., Adjustment for heterogeneity of variances by herd production level in dairy cow and sire evaluation, J. Dairy Sci. 73 (1990) 503-512.

[2] Brotherstone S., Hill W.G., Heterogeneity of variance amongst herds for milk production, Animal Prod. 42 (1986) 297-303.

[3] Costa e Silva J., Wellendorf H., Borralho N.M.G., Prediction of breeding values and expected genetic gains in diameter growth, wood density and spiral grain from parental selection in Picea abies (L.) Karst, Silvae Genet. 49 (2000) 101-109.

[4] Costa e Silva J., Borralho N.M.G., Potts B.M., Additive and nonadditive genetic parameters from clonally replicated and seedling progenies of Eucalyptus globulus, Theor. Appl. Genet. 108 (2004) 1113-1119.

[5] Dieters M.J., White T.L., Hodge G.R., Genetic parameter estimates for volume from full-sib tests of slash pine (Pinus elliottii), Can. J. For. Res. 25 (1995) 1397-1408.

[6] Dodenhoff J., Swalve H.H., Heterogeneity of variances across regions of northern Germany and adjustment in genetic evaluation, Livest. Prod. Sci. 53 (1998) 225-236.

[7] Garrick D.J., Van Vleck L.D., Aspects of selection for performance in several environments with heterogeneous variances, J. Animal Sci. 65 (1987) 409-421.

[8] Gianola D., On selection criteria and estimation of parameters when the variance is heterogeneous, Theor. Appl. Genet. 72 (1986) 671-677.

[9] Gilmour A.R., Thompson R., Cullis B.R., Average information REML, an efficient algorithm for variance parameter estimation in linear mixed models, Biometrics 51 (1995) 1440-1450.

[10] Gilmour A.R., Cullis B.R., Welham S.J., Thompson R., ASREML Reference Manual, New South Wales Agriculture, Orange, Australia, 1999.

[11] Griffing B., Concept of general and specific combining ability in relation to diallel crossing systems, Aust. J. Biol. Sci. 9 (1956) $463-$ 493

[12] Henderson C.R., Sire evaluation and genetic trends, in: Proceedings of the animal breeding and genetics symposium in honour of Dr J. Lush, Champaign, Illinois, American Society of Animal Science, American Dairy Science Association, American Poultry Science Association, 1973, pp. 10-41.

[13] Henderson C.R., Applications of Linear Models in Animal Breeding, University of Guelph, Guelph, Ontario, 1984.

[14] Hill W.G., On selection among groups with heterogeneous variance, Animal prod. 39 (1984) 473-477.
[15] Hodge G.R., Volker P.W., Potts B.M., Owen J.V., A comparison of genetic information from open-pollinated and control-pollinated progeny tests in two eucalypt species, Theor. Appl. Genet. 92 (1996) 53-63.

[16] Jordan G.J., Dutkowski G.W., Potts B.M., MacDonald A.C., Tilyard P., Borralho N.M.G., Genetic variation in North Forest Products' Eucalyptus globulus ssp. globulus base population trials, CRC-SPF Technical Report 8, Hobart, Australia, 1998.

[17] Libby W.J., Jund E., Variance associated with cloning, Heredity 17 (1962) 533-540.

[18] Lopez G.A., Potts B.M., Dutkowski G.W., Apiolaza L.A., Gelid P.E., Genetic variation and inter-trait correlations in Eucalyptus globulus base population trials in Argentina, For. Genet. 9 (2002) 217-232.

[19] Lynch M., Walsh B., Genetics and Analysis of Quantitative Traits, Sinauer Associates Inc., Sunderland, MA, USA, 1998.

[20] Meuwissen T.H.E., Van der Werf J.H.J., Impact of heterogeneous within herd variances on dairy cattle breeding schemes: a simulation study, Livest. Prod. Sci. 33 (1992) 31-41.

[21] Meuwissen T.H.E., De Jong G., Engel B., Joint estimation of breeding values and heterogeneous variances of large data files, J. Dairy Sci. 79 (1996) 310-316.

[22] Mullin T.J., Park Y.S., Estimating genetic gains from alternative breeding strategies for clonal forestry, Can. J. For. Res. 22 (1992) $14-23$.

[23] Patterson H.D., Thompson R., Recovery of interblock information when blocks sizes are unequal, Biometrika 31 (1971) 100-109.

[24] Stonecypher R.W., McCullough R.B., Estimates of additive and non-additive variances from a clonal diallel of Douglas-fir Pseudotsuga menziesii (Mirb.) Franco, in: Proceedings IUFRO Joint Meeting of Working Parties on Breeding Theory, Progeny Testing and Seed Orchards, Williamsburg, VA, USA, 13-17 October, 1986, pp. 211-227.

[25] Stram D.O., Lee J.W., Variance components testing in the longitudinal mixed effects setting, Biometrics 50 (1994) 1171-1177.

[26] Van der Werf J.H.J., Meuwissen T.H.E., De Jong G., Effects of correction for heterogeneity of variance on bias and accuracy of breeding value estimation for Dutch dairy cattle, J. Dairy Sci. 77 (1994) 3174-3184.

[27] Visscher P.M., Thompson R., Hill W.G., Estimation of genetic and environmental variances for fat yield in individual herds and an investigation into heterogeneity of variance between herds, Livest. Prod. Sci. 28 (1991) 273-290.

[28] Visscher P.M., Hill W.G., Heterogeneity of variance and dairy cattle breeding, Animal Prod. 55 (1992) 321-329.

[29] Wei X., Borralho N.M.G., Genetic control of wood basic density and bark thickness and their relationships with growth traits of Eucalyptus urophylla in south east China, Silvae Genet. 46 (1997) 245-250.

[30] Wei X., Borralho N.M.G., Genetic control of growth traits of Eucalyptus urophylla S.T. Blake in south east China, Silvae Genet. 47 (1998) 158-165.

[31] Weigel K.A., Lawlor T.J., Adjustment for heterogeneous variance in genetic evaluations for conformation of United States Holsteins, J. Dairy Sci. 77 (1994) 1691-1701.

[32] White T.L., Hodge G.R., Predicting Breeding Values with Applications in Forest Tree Improvement, Kluwer Academic Publishers, Dordrecht, 1989. 